\title{
Yeast two-hybrid screening using constitutive-active caspase-7 as bait in the identification of PA28 $\gamma$ as an effector caspase substrate
}

\author{
R Araya ${ }^{1,2}$, R Takahashi ${ }^{*, 1}$ and Y Nomura ${ }^{2}$ \\ ${ }^{1}$ Laboratory for Motor System Neurodegeneration, RIKEN Brain Science \\ Institute (BSI), Saitama 351-0198, Japan \\ 2 Department of Pharmacology, Graduate School of Pharmaceutical Sciences, \\ Hokkaido University, Sapporo 060-0812, Japan \\ * Corresponding author: Ryosuke Takahashi, Laboratory for Motor System \\ Neurodegeneration, RIKEN Brain Science Institute (BSI), 2-1 Hirosawa, Wako \\ City, Saitama 351-0198, Japan. Tel: +81-48-467-6072; \\ Fax: +81-48-462-4796; E-mail: ryosuke@brain.riken.go.jp
}

Received 10.7.01; accepted 21.8.01

Edited by $\mathrm{H}$ Ichijo

\begin{abstract}
Caspase-3 and -7 represent executioner/effector caspases that directly cause apoptotic morphological changes by cleaving various death substrates. The substrates for caspases generally interact with active caspases, but not with inactive zymogens of caspase or procaspases. Here, to isolate proteins that interact with caspase-7, we established a yeast two-hybrid screening system using reversedcaspase-7, a constitutive active mutant of caspase-7 as a bait plasmid. Screening of an adult brain cDNA library led to isolation of proteasome activator 28 subunit, PA28 $\gamma$. In vitro translates of PA28 y were cleaved by both recombinant caspase-3 and -7 . Mutagenesis of potential cleavage site $\mathrm{DGLD}^{80}$ to $\mathrm{EGLE}^{80}$ completely abolished caspase-mediated cleavage. Moreover, endogenous PA28 $\gamma$ was cleaved during not only Fas-induced apoptosis of HeLa cells, but also cisplatin-induced cell death of MCF7 cells, which are devoid of caspase-3. These findings indicate that PA28 $\gamma$ is an endogenous substrate for caspase- 3 and -7 and that yeast two-hybrid screening using reversed-caspase is a novel and useful approach to clone substrates for effector caspases. Cell Death and Differentiation (2002) 9, 322-328. DOI: 10.1038/ sj/cdd/4400949
\end{abstract}

Keywords: apoptosis; caspase-7; caspase-3; reversed-caspase7; yeast two-hybrid; PA28\%; MCF7

Abbreviations: rev-caspase, reversed caspase; rev-caspase-7-C/ $\mathrm{S}$, the active site mutant of reversed caspase-7; PA28 $\gamma$, proteasome activator 28 subunit 3; XIAP, X-linked inhibitor of apoptosis protein; zVAD-fmk, benzyloxycarbonyl-Val-Ala-Asp-fluoromethyl ketone; zDEVD-fmk, benzyloxycarbonyl-Asp-Glu-Val-Asp-fluoromethyl ketone

\section{Introduction}

Apoptosis is an actively performed cellular suicidal process essential for the development and maintenance of tissue homeostasis of multi-cellular organisms. Apoptosis is characterized by prominent morphological features such as membrane blebbing, nuclear condensation, DNA fragmentation and apoptotic body formation. A family of cysteine proteases called caspases plays a critical role in the execution of apoptosis, from nematodes to humans. To date, 14 mammalian caspases have been identified. ${ }^{1}$ Caspases are synthesized as inactive zymogens that become activated by cleavage after a specific aspartate residue within a linker domain between a large subunit (LS) and a small subunit (SS), in addition to cleaving off of the $\mathrm{NH}_{2}$-terminal prodomain. The LS and SS subsequently combine into a heterodimer to form active caspases. ${ }^{2}$ When cells receive death signals, a subset of caspases are activated in an organized fashion and lead to processing and activation of crucial effector/executioner caspases.

Effector caspases, represented by caspase- $3,-6$ and -7 , specifically cleave cellular proteins or 'death substrates', and govern the changes characterizing apoptotic morphology. Thus, death substrates play an important role in determining the final apoptotic phenotype. Caspase-dependent cleavage inactivates a subgroup of substrates such as PARP, nuclear lamin, Bcl-2 and $\beta$-catenin, whereas activates a different subgroup of substrates including MEKK1, p21-activated kinase, protein kinase $\mathrm{C} \delta$ and the ICAD/CAD complex. ${ }^{3}$ Although the role of death substrate cleavage in the apoptotic phenotype is partially understood, the substrates essential for causing cell death remain unknown.

Caspases-3 and -7 are closely related caspases in sequence similarity as well as in substrate specificity. ${ }^{4,5}$ Recombinant large or small subunit of caspase-7 can form active heteromeric complexes with the caspase-3 counterpart. ${ }^{4}$ Both caspases show overlapping substrate specificities with special preference for the DEVD motif. However, these highly related caspases are differentially regulated in subcellular localization during apoptosis, suggesting the presence of caspase- 3 or -7 specific substrates. ${ }^{6}$ The role of caspase-7 is still ill-defined, unlike caspase-3 which has been shown to play a central role in apoptosis execution. Thus, we attempted to clone new substrates for caspase-7.

Recently, Srinivasula et al succeeded in generating a constitutive active recombinant caspase- 3 and -6 by rearrangement of their subunits. ${ }^{7}$ These arranged forms of caspases were designated 'reversed-caspases' (rev-caspases), because the order of large and small subunits of these caspases are switched and reversed. Based on this technology, we devised rev-caspase-7, which is also constitutively active. Using an active-site mutant of revcaspase-7 as bait, we performed yeast two-hybrid screen- 
ing, resulting in the cloning of 205 proteasome activator 28 subunit 3, PA28 $\gamma$. Subsequent studies showed that PA28 $\gamma$ is cleaved by caspase- 3 or -7 both in vitro and in vivo, indicating that PA28 $\gamma$ is a new member of endogenous substrates for these effector caspases.

\section{Results}

\section{Production of rev-caspase-7}

Caspases are synthesized as inactive single-polypeptide zymogens and must be cleaved between the $\mathrm{NH}_{2}$-terminal of the large subunit (LS) and the $\mathrm{COOH}$-terminal of the small subunit (SS) to be activated. Effector caspases, namely caspase-3, -6 and -7, cannot undergo autocatalytic processing and have an absolute requirement for 'initiator' caspases such as caspase- 8 and -9 for processing. ${ }^{2}$

Srinivasula et al, based on the three-dimensional structure of active caspase-3, engineered contiguous caspase- 3 and -6 molecules in which the SS was fused in-frame N-terminal to the LS, and a cleavage site (i.e., DEVDG in the case of caspase-3) was introduced between the two subunits ${ }^{7}$ (Figure 1A). In contrast to their wild-type counterparts, these engineered rev-caspases, in which SS precedes LS, were able to undergo antocatalysis in an in vitro translation reaction and potently induced apoptosis when over expressed in cells, indicating that rev-caspase-3 and -6 spontaneously fold into active conformation.

Because caspase-3 and caspase-7 are highly related molecules, we constructed rev-caspase-7 along with caspase-3 using essentially the same strategy as Srinivasula and his coworkers (Figure 1A). First, we examined whether wild-type procaspases and rev-caspases undergo autocatalytic processing in an in vitro translation reaction (Figure 1B). As expected, rev-caspase-3 and -7 (Rev) were able to undergo processing into LS and SS in an in vitro system, whereas wild-type caspase-3 and -7 (WT) were not.

Moreover, the processing was completely inhibited by mutating the active site cysteine to serine of rev-caspase-3 and -7 (Rev-C/S), indicating that rev-caspase-3 and -7 are catalyzed by their own activities (Figure 1B, lanes 3 and 6).

\section{Rev-caspase-7 retains activity in yeast}

Given that rev-caspase-7-folded into active conformation in an in vitro translation system, we decided to clone molecules that interact with caspase-7, including substrates and inhibitors, using a yeast two-hybrid screening with revcaspase-7 as the bait. To determine whether rev-caspase-7 was also autoprocessed in yeast, we introduced cDNAs for wild-type procaspase-7, rev-caspase-7 and active site cysteine to serine mutant of rev-caspase-7 into the yeast two-hybrid vector pGilda. In this vector, cDNAs are expressed as $\mathrm{NH}_{2}$-terminal fusion proteins with the LexA DNA binding domain under control of the inducible GAL1 promoter. The expression of caspase-7 constructs was induced by incubation with galactose for $12 \mathrm{~h}$ and checked by Western blotting (Figure 2). Similar to the in vitro translation experiments, revcaspase-7 (Rev) was auto-processed to produce the large subunit (LS), whereas the active site mutant of rev-caspase-7 (Rev-C/S) was not. Interestingly, unlike the in vitro translation product, procaspase-7 (WT) was partially cleaved. This processing is most likely caused by autocatalysis induced by artificial massive expression of recombinant procaspase-7 in yeast. Moreover, expression of rev-caspase-7 in yeast, but not that of procaspase-7 or active site mutant of rev-caspase7, impaired cell growth, consistent with the previous finding that caspase-3 expression induces growth inhibition in an activity-dependent manner ${ }^{8}$ (data not shown).

\section{Rev-caspase-7 and rev-caspase-7-C/S bind to natural caspase inhibitors in yeast}

Baculovirus protein p35 and human inhibitor of apoptosis proteins (IAPS), XIAP, c-IAP1, c-IAP2 and survivin have been shown to tightly bind to and inhibit active caspase-7 but not inactive procaspase-7..$^{9-12}$ Thus, we examined whether revcaspase-7 also bound to these natural caspase inhibitors in yeast to obtain evidence that rev-caspase-7 behaves similarly to active caspase-7. Yeasts were co-transformed with galactose-inducible pGilda-wild-type procaspase-7, -rev-caspase-7 or -rev-caspase-7-C/S and a galactose-inducible pJG4-5 vector encoding cDNAs for XIAP, c-IAP1, c-IAP2 or
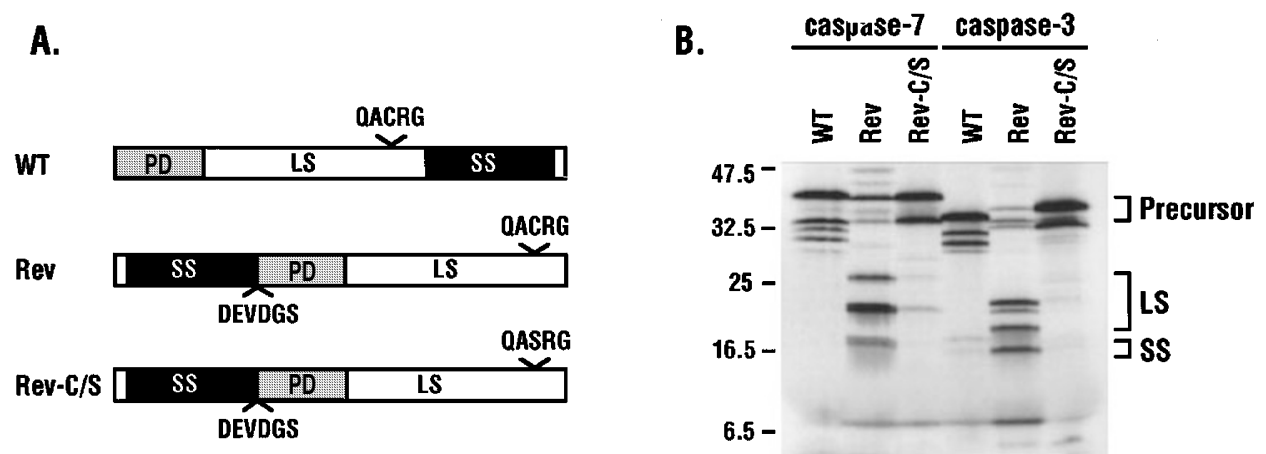

Figure 1 A construction of rev-caspase-7 mutants (A) and autoprocessing of rev-caspase-3 and -7 in an in vitro translation reaction (B). (A) PD, LS, SS stands for prodomain, large subunit and small subunit, respectively. QACRG, QASRG and DEVDGS indicates the sequence surrounding active-site residue cysteine and mutated residue serine and the sequence between SS and PD, respectively. (B) Caspase-3 and -7 (WT) or their rev-versions (Rev), including active site Cys to Ser mutants (Rev-C/S) in pcDNA3 constructs were in vitro translated in the presence of $\left[{ }^{35}\right.$ S]methionine. The translation products were then analyzed by SDS-PAGE and autoradiography. The LS and SS are indicated 
survivin. As shown in Figure 3, rev-caspase-7 bound to XIAP, but not to c-IAP1, c-IAP2, or p35, whereas wild-type procaspase-7 did not bind to XIAP, as expected. The growth of yeasts expressing rev-caspase-7 was generally inhibited, except for the one strain that expressed both rev-caspase-7 and XIAP (data not shown). On the other hand, rev-caspase7-C/S bound to p35, in addition to XIAP. The growth of yeasts expressing rev-caspase-7-C/S was not impaired. These results indicate that rev-caspase-7 binds to at least some caspase inhibitors in yeasts. Neither rev-caspase-7 nor revcaspase-7-C/S mutant bound to C-IAP1, c-IAP2 or survivin, most likely because the binding affinities of these IAPs for caspase-7 are much lower than those of XIAP. ${ }^{11,13}$ Moreover, the inhibitory activity of p35 may not be strong enough to completely inhibit the activity of rev-caspase-7 in yeast, thus leading to growth arrest and a negative binding result. Based on these findings, we decided to use rev-caspase-7-C/S as bait in the screening of a yeast two-hybrid cDNA library in order to clone active caspase-7 interacting proteins, including

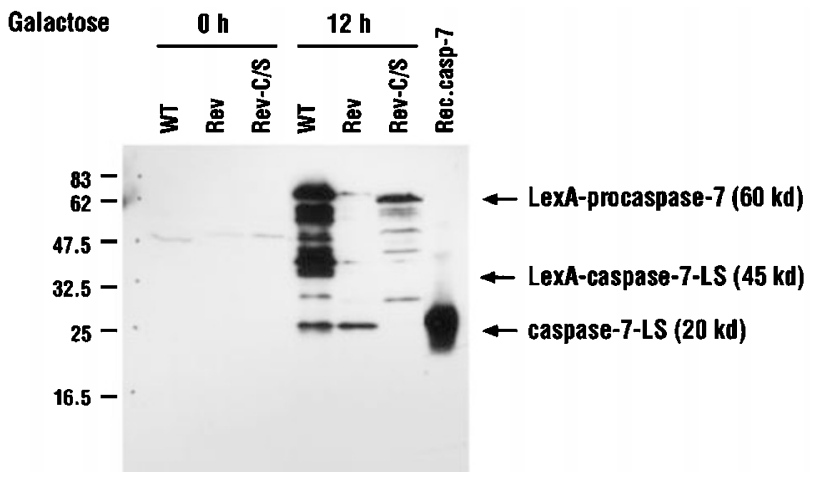

Figure 2 Expression of rev-caspase-7 mutants in yeast. EGY48 yeast cells were transformed with pGilda expression plasmids carrying indicated caspase7 mutants, and were grown overnight in SD-Trp, His, Ura liquid media containing $2 \%$ glucose. Cells were centrifuged and the cell pellet washed with sterile water. Cells were diluted to an $\mathrm{OD}_{600}$ of $1.0 \mathrm{in} 120 \mathrm{ml}$ of fresh SD-Trp, His, Ura liquid media containing $2 \%$ galactose- $1 \%$ raffinose to induce expression of caspase-7. After $12 \mathrm{~h}$ culture, total yeast lysates $(50 \mu \mathrm{g} / \mathrm{lane})$ were subjected to immunoblotting using anti-caspase-7 monoclonal antibody. $0.1 \mathrm{nM}$ of purified recombinant caspase- 7 was used as the positive control substrates and inhibitors such as IAP or p35 related molecules.

\section{Substrate candidates for caspase-7 were identified by yeast two-hybrid screening}

We used pGilda-rev-caspase-7-C/S as a bait plasmid to screen a mouse adult brain cDNA library. A total of 110 positive clones were obtained by screening $2 \times 10^{6}$ transformants. Partial sequencing of positive clones yielded 20 candidate molecules for caspase-7 interacting protein. To examine whether these clones were substrates for caspase7 , in vitro translates of these clones were produced and incubated with recombinant caspases. As shown in Figure $4 \mathrm{~A}$, four of the 20 candidates were cleaved by recombinant caspases, suggesting that these proteins were caspase substrates. Clone \#15 was cleaved only by caspase-7, whereas clones \#52, \#77, and \#105 were cleaved by both caspase- 3 and -7 and also by caspase- 6 to a lesser extent. Moreover, these four clones were cleaved by recombinant caspases in a dose dependent manner (data not shown). These results indicate that this system is highly effective in identifying caspase substrates.

\section{PA28 was cleaved by caspase-3 and $\mathbf{- 7}$ in vitro}

Of the substrate candidates, we focused on clone \#105, which encoded mouse proteasome activator 28 subunit 3 (PA28 $\gamma$ ), as evolutionarily it is a very well conserved protein from drosophila to humans and appears to have a biologically important role. ${ }^{14}$

First, we cloned full length human PA28 $\gamma$ cDNA and confirmed that human PA28 $\gamma$ was cleaved by recombinant caspase-3, -7 and also by caspase- 6 to a lesser extent in vitro, consistent with partial clone (\#105) cleavage (Figure $4 \mathrm{~B}$ and data not shown). These results suggest that the cleavage site for caspases is conserved between human and mouse. In the human PA28 $\gamma$ sequence, we found a $D G L D^{80} G$ sequence, which was conserved in the corresponding sequence of mouse PA28 $\gamma$ and very well fitted the optimal tetrapeptide recognition sequence for caspase3 or -7 (DEXD). ${ }^{15}$ To examine whether this sequence was
caspase-7-WT

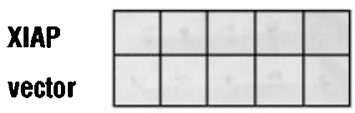

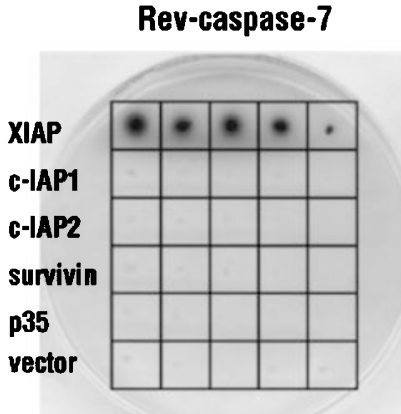

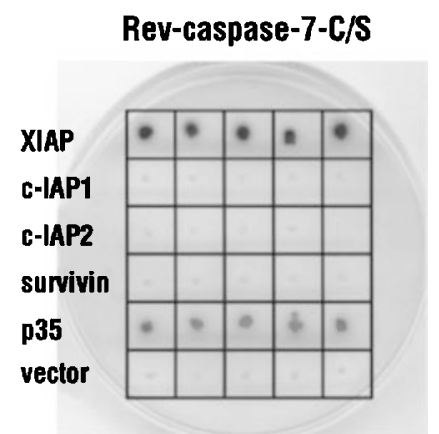

Figure 3 Binding assay of caspase-7-WT and rev-caspase-7 mutants to IAP family and p35 in yeast two-hybrid system. EGY48 yeast cells were transformed with pGilda plasmids carrying each caspase-7 mutant and pJG4-5 plasmids carrying various IAP family members (XIAP, C-IAP1, c-IAP2, or survivin) or baculovirus p35 or no insert (vector) and pSH18-34 plasmids carrying the lacZ reporter gene. Each of five transformants was spotted from selective glucose-containing medium onto selective gal/raf- inducing medium containing $80 \mu \mathrm{g} / \mathrm{ml}$ of X-gal 
the cleavage site, we constructed two PA28 $\gamma$ mutants: one with Asp-80 to Glu (DGLE ${ }^{80}$ ), named PA-DE, and the other with Asp-77 and -80 to Glu $\left(E_{G L E}{ }^{80}\right)$, named PA-EE. These mutants, PA-DE and PA-EE, were not cleaved by recombinant caspases, indicating that the caspase-3/-7 and caspase- 6 cleavage site of human PA28 $\gamma$ was Asp- 80 (Figure 4B and data not shown).

\section{PA28 $\gamma$ was cleaved by caspase-7 in vivo}

To examine the cleavage of PA28 $\gamma$ by caspase-7 in vivo, HEK 293T cells were co-transfected with HA-tagged caspase $-7 \Delta \mathrm{N}$ and caspase- $7 \Delta \mathrm{N}-\mathrm{C} / \mathrm{S}$, of which the $\mathrm{NH}_{2}-$ terminal 23 amino acids are deleted, and $\mathrm{NH}_{2}$-terminal Flagtagged PA28 $\gamma$. Unlike full-length procaspase-7, caspase$7 \Delta \mathrm{N}$ was processed and induced cell death when overexpressed in mammalian cells (data not shown). As shown in Figure 5, PA-WT was cleaved by caspase- $7 \Delta \mathrm{N}$, generating an $11 \mathrm{kDa} N$-terminal fragment, but not by caspase- $7 \Delta \mathrm{N}-\mathrm{C} /$ $\mathrm{S}$. The PA-EE mutant was not cleaved at all by caspase- $7 \Delta \mathrm{N}$ or caspase-7 $\Delta \mathrm{N}-\mathrm{C} / \mathrm{S}$. Moreover, Flag-tagged PA28 $\gamma$ was coimmunoprecipitated with the rev-caspase-7-C/S mutant after both plasmids were co-transfected into HEK293T cells, and the PA-EE mutant, unlike the wild type, hardly bound to revcaspase-7-C/S (data not shown). These data indicate that PA28 $\gamma$ interacts with and is cleaved by caspase-7 at Asp-80 in vivo, in exactly the same way as in an in vitro cleavage experiment.

A.

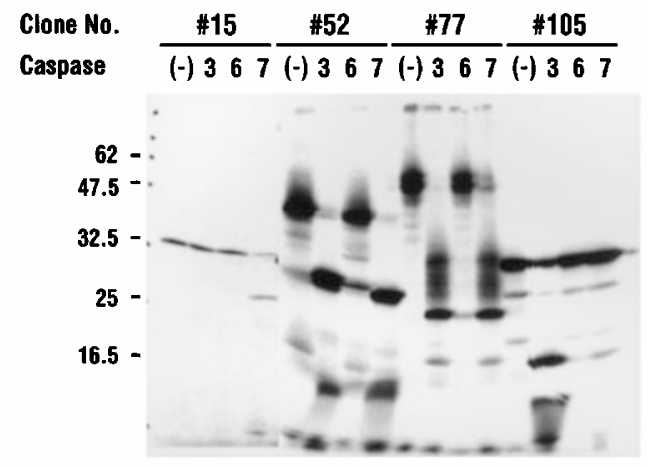

B.

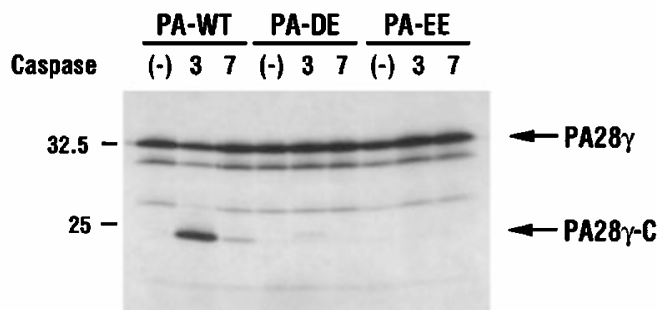

Figure 4 In vitro cleavage assay of positive clones by recombinant caspases. Each clone was in vitro translated in the presence of $\left[{ }^{35} \mathrm{~S}\right]$ methionine. The translated products were incubated with recombinant caspases for $1 \mathrm{~h}$, and then analyzed by SDS-PAGE and autoradiography. (A) \#15 was cleaved only by caspase-7 whereas \#52, \#77, \#105 were cleaved not only by caspase- 3 and -7 but also caspase- 6 to a lesser extent. (B) Wildtype human PA28 $\gamma$ DGLE $^{80}$ (PA-WT) was cleaved by caspases, whereas PA28y mutants DGLE ${ }^{80}(\mathrm{PA}-\mathrm{DE})$ and $\mathrm{EGLE}^{80}$ (PA-EE) were not. The Cterminal fragment of PA28 $\gamma$ is denoted PA28 $\gamma-\mathrm{C}$

\section{PA28 $\gamma$ is an endogenous substrate of caspase-3/-7 like caspases}

Next, we generated anti-serum against the C-terminal portion of PA28 $\gamma$ (81-92 a.a.), and checked the cleavage of endogenous PA28 $\gamma$ during Fas-induced apoptosis of HeLa $\mathrm{K}$ cells. As shown in Figure 6, PA28 $\gamma$ was cleaved with a

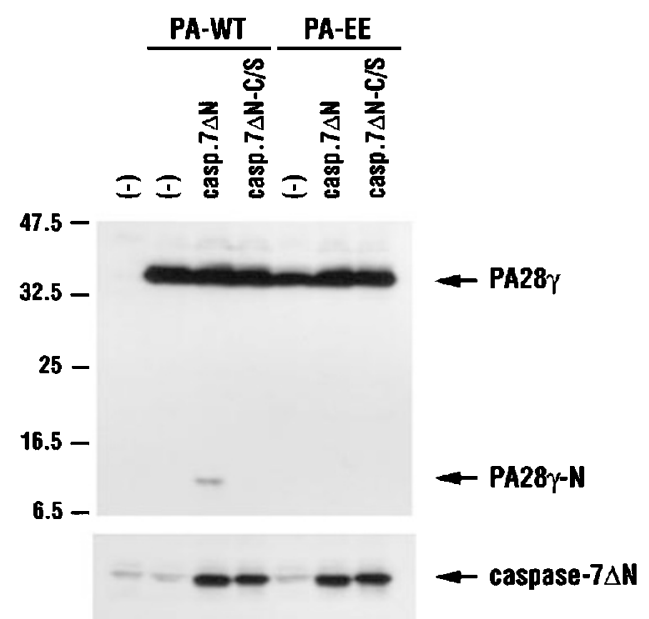

Figure 5 In vivo cleavage of PA28 $\gamma$ by caspase- $7 \Delta \mathrm{N}$ in mammalian cells 293T cells were co-transfected with HA-tagged caspase-7 $\mathrm{N}$ (in which the $\mathrm{N}$ terminal 23 amino acids were deleted), and Flag-tagged PA28 $\gamma$. At $48 \mathrm{~h}$ after transfection, total cell lysates were prepared and then subjected to immunoblotting using anti-Flag monoclonal antibody and anti-caspase-7 polyclonal antibody. The N-terminal fragment of PA28 $\gamma$ is denoted PA28 $\gamma-\mathrm{N}$

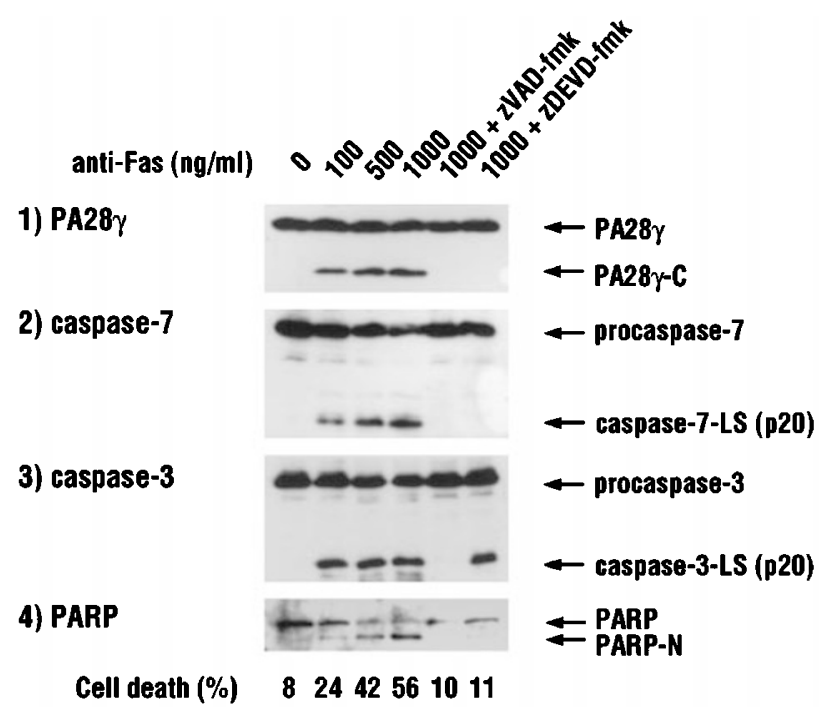

Figure 6 PA28 $\gamma$ was cleaved during Fas-induced apoptosis by caspase-3/-7 like caspases. HeLa K cells were treated with anti-Fas monoclonal antibody at the indicated dose with or without $50 \mu \mathrm{M}$ of z-DEVD-fmk or z-VAD-fmk. At $12 \mathrm{~h}$ after treatment, total cell lysates were prepared and then subjected to immunoblotting using anti-PA28 $\gamma$ polyclonal antibody (1), anti-caspase-7 monoclonal antibody (2), anti-caspase-3 polyclonal antibody (3) and antiPARP monoclonal antibody (4). PA28 $\gamma$ was cleaved in a dose dependent manner accompanying caspase- 7 and -3 activation, and the cleavage was blocked by caspase inhibitors 
similar time course to that of the cleavage of caspase-7 or -3 during apoptosis. Moreover, the cleavage was blocked by zVAD-fmk, an irreversible pan-caspase peptide inhibitor, and zDEVD-fmk, an irreversible peptide inhibitor for caspase-3/-7 like caspases. Although the cleavage of caspase- 3 was not inhibited by zDEVD-fmk treatment, caspase-3 activity was blocked, and therefore PARP, the substrates of caspase-3 and -7, was not cleaved at all. Like PARP, PA28 $\gamma$ was not cleaved by caspase-3 and -7 upon zDEVD-fmk treatment. These findings indicate that PA28 $\gamma$ is directly cleaved by caspase-7, -3 , or closely related caspases in vivo.

Finally we examined whether endogenous PA28 $\gamma$ is cleaved during the apoptosis of MCF-7 cells, which are devoid of caspase-3 owing to a functional deletion of the caspase-3 gene. ${ }^{16}$ Despite the lack of caspase-3, this cell line shows sensitivity to various death stimuli, providing an excellent system with which to test whether caspase-3 is indispensable for the cleavage of a specific death substrate. ${ }^{17}$ We induced apoptosis in MCF7 cells with addition of cisplatin and checked whether PA28 $\gamma$ was cleaved in apoptotic MCF7 cells. Very similar to anti-Fas antibody-treated HeLa K cells, PA28 $\gamma$ was cleaved with the concomitant processing of caspase-7, and the cleavage was inhibited by zVAD-fmk and zDEVD-fmk. These results indicate that endogenous PA28 $\gamma$ in MCF7 cells is cleaved by a DEVD-specific caspase other than caspase-3, most likely caspase-7.

\section{Discussion}

The yeast two-hybrid system is a versatile method with which to systematically identify interacting proteins. In the present study, we used this system to clone caspase substrates using genetically engineered rev-caspase-7, and successfully identified PA28 $\gamma$ as a substrate for caspase-3 or -7 .

Previous to our study, Kamada et al developed a method of cloning the genes of caspase substrates using a modified yeast two-hybrid system, and identified gelsolin as a substrate of caspase-3. ${ }^{18}$ They expressed large and small subunits separately using $\mathrm{ADH} 1$ promoter, which ensured an equimolar ratio of these two subunits. Compared to their method, our method of adopting an active site mutant of rev-caspase as bait enables utilization of any commercially available yeast two-hybrid vector and appears to be more convenient. On the other hand, unlike the Kamada et al method, in our procedure an active site mutant of rev-caspase-7 (rev-caspase-7-C/S) remains a single polypeptide due to the lack of autocatalysis, raising the possibility that this mutant does not have the proper structure of active caspase. We addressed this concern by demonstrating specific binding between rev-caspase-7-C/S and natural caspase inhibitors including p35 and XIAP. Recent structural studies have demonstrated that both inhibitors bind to the active site of caspase-7, indicating that rev-caspase-7-C/S retains a substrate binding groove identical to that of active caspase-7. ${ }^{19-22}$ Since revcaspase- 3 and -6 have been engineered and shown to have the active form conformation, substrates for these caspases could also be systematically cloned using yeast two-hybrid screening with rev-caspases-C/S. ${ }^{7}$
In the present study, we have identified PA28 $\gamma$ as a substrate for caspase-3 or -7. PA28 $\gamma$ was originally termed $\mathrm{Ki}$ antigen, which was initially identified using autoantibodies found in sera of patients with systemic lupus erythematosus. ${ }^{23}$ Subsequent studies revealed that $\mathrm{Ki}$ antigen has sequence similarity to proteasome activators $\mathrm{PA} 28 \alpha$ and PA28 $\beta$, and was renamed PA28 $\gamma .{ }^{24} \mathrm{PA} 28 \alpha$ and PA28 $\beta$ assemble into a heteromultimer called PA28, which has been shown to associate with and greatly stimulate multiple peptidase activities of the $20 \mathrm{~S}$ proteasome. PA28 is upregulated by stimulation with IFN- $\gamma$ and thought to be involved in the generation of $\mathrm{MHC}$ class I ligands. ${ }^{25}$ In contrast, the physiological role of PA28 $\gamma$ is unclear. Mice deficient in the PA28 $\gamma$ gene are born without detectable developmental abnormalities. ${ }^{26}$ However, mild growth retardation of $\mathrm{PA} 28 \gamma^{-1-}$ compared with $\mathrm{PA} 28 \gamma^{+-}$or $\mathrm{PA} 28 \gamma^{+/+}$mice has been observed. Moreover, embryonic fibroblasts derived from PA28 $\gamma^{-1-}$ mice displayed a slower growth rate than those from wild-type mice, indicating that PA28 $\gamma$ is involved in cell proliferation. Since PA28 $\gamma$ localizes almost exclusively in the nucleus, it is speculated to play a role in the degradation of nuclear proteins regulating cell cycle progression. ${ }^{26,27}$ To examine the functional significance of the cleavage of PA28 $\gamma$ during apoptosis, we observed the effect of over-expression of $\mathrm{PA} 28 \gamma \mathrm{EE}$, a caspase-uncleavable mutant of PA28 $\gamma$, on apoptosis (refer to Figure 4). However, over-expression of PA28 $\gamma$ EE did not affect either the time course or cell morphology during apoptosis of HEK 293 cells and $\mathrm{SH}$ SY5Y cells induced by various stimuli including Fas overexpression and tunicamycin treatment, respectively ${ }^{12,28}$ (data not shown). Further study is required to clarify the significance of caspase-3/-7-mediated cleavage of PA28 $\gamma$.

In conclusion, we have succeeded in identifying a novel substrate for caspase-3 or -7 using a yeast two-hybrid screening with rev-caspase-7 as bait. Systematic cloning of more caspase substrates using this convenient method will further elucidate the roles of caspases and their substrates in apoptosis.

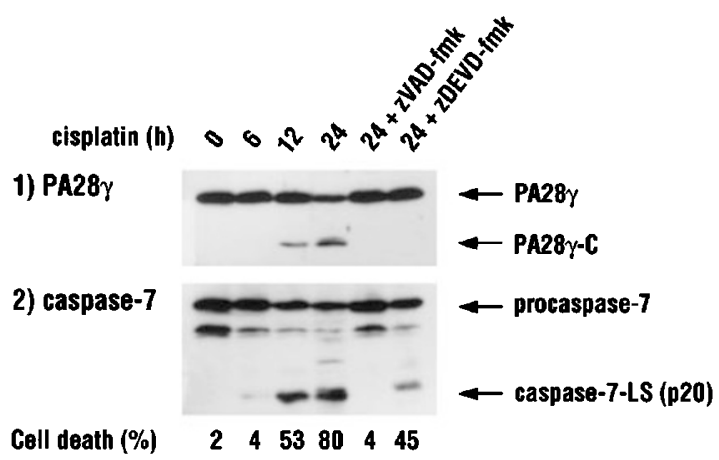

Figure 7 PA28 $\gamma$ was cleaved during cisplatin induced apoptosis by a DEVDspecific caspase other than caspase-3. MCF7 cells were treated with $100 \mu \mathrm{M}$ of cisplatin for the indicated time with or without $50 \mu \mathrm{M}$ of zDEVD-fmk or zVADfmk. Total cell lysates were prepared and then subjected to immunoblotting using anti-PA28 $\gamma$ polyclonal antibody (1) and anti-caspase-7 monoclonal antibody (2). PA28 $\gamma$ was cleaved in a time dependent manner accompanying caspase- 7 activation, and the cleavage was blocked by caspase inhibitors 


\section{Material and Methods}

\section{Reagents, cell culture and transfection}

Benzyloxycarbonyl-Val-Ala-Asp-fluoromethyl ketone (zVAD-fmk) and benzyloxycarbonyl-Asp-Glu-Val-Asp-fluoromethyl ketone (zDEVDfmk) were purchased from Peptide Institute (Osaka, Japan) and Enzyme Systems Products (Livermore, CA, USA).

HEK293T, HeLa K cells were kind gifts of Dr. S Yonehara and maintained in Dublecco's modified Eagle's medium (DMEM) supplemented with $10 \%$ fetal bovine serum at $37^{\circ} \mathrm{C}$ in humidified $5 \%$ $\mathrm{CO}_{2} / 95 \%$ air. HEK293T cells were transfected using Lipofectamine PLUS ${ }^{T M}$ reagent (Gibco BRL, Grand Island, NY, USA) according to the manufacturer's instructions. MCF-7 cells were obtained from ATCC (Rockville, MD, USA) and maintained in DMEM supplement with $10 \%$ fetal bovine serum at $37^{\circ} \mathrm{C}$ in humidified $5 \% \mathrm{CO}_{2} / 95 \%$ air.

\section{Construction of rev-caspase mutants}

cDNAs encoding rev-caspase precursors were generated by PCR. Rev-caspase-3 was constructed as previously described. ${ }^{7}$

To construct rev-caspase-7, the large and small subunits of caspase-7 were amplified with the following primers using the caspase-7 cDNA as a template: LS-forward, CCGGATCCATGGCAGATGATCAGGGCTGTATT; LS-reverse, CTGCTCGAGTTAGTCGGCCTGGATGCCATCATC; sS-forward, GGAATTCTCGGGGCCCATCAATGACACA; SS-reverse, CCGGATCCATCAACTTCATCTTGACTGAAGTAGAGTTC. The LS PCR product was excised with BamHI/Xhol, while SS was excised with EcoRI/BamHI. These products were then simultaneously subcloned into the $E c o R I / X h o l$ site of the mammalian expression vector pcDNA3 or that of the yeast expression vector $p$ Gilda.

\section{Yeast transformation and characterization}

The yeast strain EGY48 ((lexA operators) ${ }_{6}$-LEU2) was maintained on an appropriate dropout medium to select for plasmid marker expression. Transformations were performed using the LiOAc method using $1 \mu \mathrm{g}$ of each plasmid (pGilda, pJG4-5, pSH18-34).

For cDNA library screening, EGY48 cells containing pGilda and pSH18-34 were grown in glucose containing synthetic dropout liquid medium lacking histidine and uracil (SD- His, Ura). The cells were then transformed with $125 \mu \mathrm{g}$ of mouse adult brain cDNA library using the LiOAc method. Transformants were plated onto galactose/raffinose, $X$ gal-containing SD-Trp, His, Ura plates which lacks tryptophan in addition to histidine and uracil. An aliquot of transformants was also plated onto glucose-containing SD-Trp, His, Ura plates to determine transformation efficiency. The blue colonies on galactose/raffinose, $\mathrm{X}$ gal-containing SD-Trp, His, Ura plates were spotted onto galactose/ raffinose, X-gal -containing SD-Trp, His, Ura, Leu plates.

\section{In vitro translation of caspases or positive yeast two-hybrid clones}

Caspases and yeast two-hybrid clones were in vitro translated in the presence of $\left[{ }^{35} \mathrm{~S}\right]$ methionine in rabbit reticulocyte lysate with a T7RNA polymerase-coupled TNT kit (Promega, Madison, WI, USA), using the pcDNA3 constructs as templates according to the manufacturer's instructions.

\section{Generation of anti-PA28 $\gamma$ polyclonal antibody}

Antibodies were raised against a region of human PA28 $\gamma$ protein spanning amino acids $81-92$, which are located immediately after the putative caspase-3/-7 cleavage site DGLD. A synthetic polypeptide
(GPTYKKRRLDEC) was cross-linked to keyhole limpet hemocyanine (KLH, Pierce, Rockford, IL, USA), emulsified in Freund's adjuvant, and used to immunize rabbits (1 $\mathrm{mg}$ in complete Freund's adjuvant for the first injection, followed every $2-3$ weeks by $1 \mathrm{mg}$ in incomplete Freund's adjuvant). Rabbit serum were divided into aliquots and kept frozen at $-20^{\circ} \mathrm{C}$.

\section{Immunoblot analyses}

The yeast cell cultures were centrifuged at $5000 \times g$ and the cell pellet washed with sterile water and resuspended in lysis buffer $(20 \mathrm{mM}$ PIPES pH 7.2, $100 \mathrm{mM} \mathrm{NaCl}, 1 \mathrm{mM}$ EDTA, $10 \%$ Sucrose, $0.1 \%$ CHAPS, $10 \mathrm{mM}$ DTT). Equal volumes of acid-washed $0.6 \mathrm{~mm}$ glass beads were added to each sample followed by vortexing for $10 \times 30 \mathrm{~s}$ with $1 \mathrm{~min}$ intervals on ice. Equal amounts of each sample $(50 \mu \mathrm{g}$ protein) were subjected to $16 \%$ SDS - PAGE followed by transfer to a PVDF membrane. The membrane was probed with anti-caspase-7 monoclonal antibody (MBL, Nagoya, Japan).

The mammalian cells $\left(5 \times 10^{5}\right)$ were washed with PBS and resuspended in lysis buffer ( $50 \mathrm{mM}$ Tris- $\mathrm{HCl}(\mathrm{pH} 7.5), 150 \mathrm{mM} \mathrm{NaCl}$, $0.5 \%$ Nonidet P-40, $1 \mathrm{mM}$ EDTA) with a protease inhibitor cocktail tablet (Roche Diagnostics, Mannheim, Germany). Equal amounts of each sample (30 $\mu \mathrm{g}$ protein) were subjected to $16 \%$ SDS-PAGE followed by transfer to a PVDF membrane. The membrane was probed with anti-PA28 $\gamma$ polyclonal antibody, anti-Flag monoclonal antibody (Sigma, St. Louis, MO, USA), anti-caspase-7 monoclonal antibody (MBL), anti-caspase-3 polyclonal antibody (a gift from Dr. Hong-Gang Wang) and anti-PARP monoclonal antibody (Clontech, Palo Alto, CA, USA).

\section{Acknowledgements}

We thank Dr. John Reed, Dr. Guy Salvesen and Dr. Paul Friesen for cDNAs for IAPs, caspases and p35, respectively. We also thank Dr. Yasuyuki Suzuki and Dr. Yuzuru Imai for valuable advice and discussion. This work was funded by research grants from RIKEN BSI, a Grant-in-Aid from the Japan Society for the Promotion of Science and grants from the Ministry of Health and Welfare, Japan.

\section{References}

1. Hu S, Snipas SJ, Vincenz C, Salvesen G and Dixit VM (1998) Caspase-14 is a novel developmentally regulated protease. J. Biol. Chem. 273: 29648-29653

2. Salvesen GS and Dixit VM (1997) Caspases: intracellular signaling by proteolysis. Cell 91: $443-446$

3. Thornberry NA and Lazebnik Y (1998) Caspases: enemies within. Science 281: $1312-1316$

4. Fernandes-Alnemri T, Takahashi A, Armstrong R, Krebs J, Fritz L, Tomaselli KJ, Wang L, Yu Z, Croce CM, Salveson G, Earnshaw WC, Litwack G and Alnemri ES (1995) Mch3, a novel human apoptotic cysteine protease highly related to CPP32. Cancer Res. 55: 6045-6052

5. Duan H, Chinnaiyan AM, Hudson PL, Wing JP, He WW and Dixit VM (1996) ICELAP3, a novel mammalian homologue of the Caenorhabditis elegans cell death protein Ced-3 is activated during Fas- and tumor necrosis factor-induced apoptosis. J. Biol. Chem. 271: 1621-1625

6. Chandler JM, Cohen GM and MacFarlane M (1998) Different subcellular distribution of caspase- 3 and caspase-7 following Fas-induced apoptosis in mouse liver. J. Biol. Chem. 273: 10815-10818

7. Srinivasula SM, AhmadM, MacFarlane M, Luo Z, Huang Z, Fernandes-Alnemri T and Alnemri ES (1998) Generation of constitutively active recombinant caspases -3 and -6 by rearrangement of their subunits. J. Biol. Chem. 273: $10107-10111$ 
8. Wright ME, Han DK, Carter L, Fields S, Schwartz SM and Hockenbery DM (1999) Caspase-3 inhibits growth in Saccharomyces cerevisiae without causing cell death. FEBS Lett. 446: $9-14$

9. Zhou Q and Salvesen GS (2000) Viral caspase inhibitors CrmA and p35. Methods Enzymol. 322: 143-154

10. Deveraux QL, Takahashi R, Salvesen GS and Reed JC (1997) X-linked IAP is a direct inhibitor of cell-death proteases. Nature. 388: 300-304

11. Roy N, Deveraux QL, Takahashi R, Salvesen GS and Reed JC (1997) The C-IAP1 and C-IAP-2 proteins are direct inhibitors of specific caspases. EMBO J. 16: 6914-6925

12. Takahashi R, Deveraux Q, Tamm I, Welsh K, Assa-Munt N, Salvesen GS and Reed JC (1998) A single BIR domain of XIAP sufficient for inhibiting caspases. J. Biol. Chem. 273: 7787-7790

13. Tamm I, Wang Y, Sausville E, Scudiero DA, Vigna N, Oltersdorf T and Reed JC (1998) IAP-family protein survivin inhibits caspase activity and apoptosis induced by Fas (CD95), Bax, caspases, and anticancer drugs. Cancer Res. 58 : $5315-5320$

14. Masson $P$, Andersson $O$, Petersen UM and Young $P$ (2001) Identification and Characterization of a Drosophila Nuclear Proteasome Regulator. A HOMOLOG OF HUMAN 11 S REGgamma (PA28gamma). J. Biol. Chem. 276: 1383-1390

15. Thornberry NA, Rano TA, Peterson EP, Rasper DM, Timkey T, Garcia-Calvo M, Houtzager VM, Nordstrom PA, Roy S, Vaillancourt JP, Chapman KT and Nicholson DW (1997) A combinatorial approach defines specificities of members of the caspase family and granzyme B. Functional relationships established for key mediators of apoptosis. J. Biol. Chem. 272: 17907-17911

16. Janicke RU, Sprengart ML, Wati MR and Porter AG (1998) Caspase-3 is required for DNA fragmentation and morphological changes associated with apoptosis. J. Biol. Chem. 273: 9357-9360

17. Janicke RU, Ng P, Sprengart ML and Porter AG (1998) Caspase-3 is required for alpha-fodrin cleavage but dispensable for cleavage of other death substrates in apoptosis. J. Biol. Chem. 273: 15540-15545

18. Kamada S, Kusano H, Fujita H, Ohtsu M, Koya RC, Kuzumaki N and Tsujimoto Y (1998) A cloning method for caspase substrates that uses the yeast two-hybrid system: cloning of the antiapoptotic gene gelsolin. Proc. Natl. Acad. Sci. USA 95: $8532-8537$
19. Xu G, Cirilli M, Huang Y, Rich RL, Myszka DG and Wu H (2001) Covalent inhibition revealed by the crystal structure of the caspase-8/p35 complex. Nature 410: $494-497$

20. Huang Y, Park YC, Rich RL, Segal D, Myszka DG and Wu H (2001) Structural basis of caspase inhibition by XIAP: differential roles of the linker versus the BIR domain. Cell 104: 781-790

21. Chai J, Shiozaki E, Srinivasula SM, Wu Q, Dataa P, Alnemri ES and Shi Y (2001) Structural basis of caspase-7 inhibition by XIAP. Cell 104: 769-780

22. Suzuki Y, Nakabayashi Y, Nakata K, Reed JC and Takahashi R (2001) XIAP inhibits caspase-3 and -7 in distinct modes. J. Biol. Chem. 18: 18.

23. Tojo T, Kaburaki J, Hayakawa M, Okamoto T, Tomii M and Homma M (1981) Precipitating antibody to a soluble nuclear antigen quot;Kiquot; with specificity for systemic lupus erythematosus. Ryumachi. 21: 129-140

24. Ahn JY, Tanahashi N, Akiyama K, Hisamatsu H, Noda C, Tanaka K, Chung CH, Shibmara N, Willy PJ, Mott JD. (1995) Primary structures of two homologous subunits of PA28, a gamma-interferon-inducible protein activator of the $20 \mathrm{~S}$ proteasome. FEBS Lett. 366: 37-42

25. Tanaka K and Kasahara M (1998) The MHC class I ligand-generating system: roles of immunoproteasomes and the interferon-gamma-inducible proteasome activator PA28. Immunol. Rev. 163: 161-176

26. Murata S, Kawahara H, Tohma S, Yamamoto K, Kasahara M, Nabeshima Y, Tanaka Kand Chiba T (1999) Growth retardation in mice lacking the proteasome activator PA28gamma. J. Biol. Chem. 274: 38211-38215

27. WojcikC, Tanaka K, Paweletz N, Naab U and WilkS (1998) Proteasome activator (PA28) subunits, alpha, beta and gamma (Ki antigen) in NT2 neuronal precursor cells and HeLa S3 cells. Eur. J. Cell. Biol. 77: 151-160

28. Imai Y, Soda M and Takahashi R (2000) Parkin suppresses unfolded protein stress-induced cell death through its E3 ubiquitin-protein ligase activity. J. Biol. Chem. 275: 35661-35664 